\title{
Molecular Interaction and Inhibitory Activity of Dandelion's Compounds on Nucleoprotein: A Therapeutic Intervention in Lassa Fever
}

\author{
Babatomiwa Kikiowo $^{1, *(\mathbb{D})}$, Ebenezer Ayomide Oni ${ }^{1}{ }^{\mathbb{D}}$, Opeyemi Iwaloye ${ }^{2} \mathbb{D}$, Olumide Kayode \\ Inyang $^{3}(\mathbb{D})$, Adebowale Abiodun Alade ${ }^{1(\mathbb{D})}$, Sunday Temitope Akinwotu ${ }^{1}$, , Oluwadunni Ruth \\ Oluwalade $^{4 \text { (iD }}$ \\ 1 Department of Biochemistry, Adekunle Ajasin University, Nigeria \\ 2 Bioinformatics and Molecular Biology Unit, Department of Biochemistry, Federal University of Technology Akure, \\ Akure, Ondo State, Nigeria \\ 3 Centre for Education Programs in Bioinformatics, Moscow Institute of Physics and Technology (MIPT), Dolgoprudny, \\ Russia \\ 4 Wesley University, Ondo State, Nigeria \\ * Correspondence: 45719155fa@aaua.edu.ng;
}

Scopus Author ID 57219288106

Received: 10.12.2020; Revised: 20.01.2021; Accepted: 24.01.2021; Published: 30.01.2021

\begin{abstract}
Lassa fever (LF) is an acute and sometimes fatal viral hemorrhagic fever caused by the Lassa virus (LASV). It is a major public health challenge and endemic exclusively in West Africa. Despite the large toll of human morbidity and mortality, no vaccine or effective drugs are available to treat this disease. Therefore, there is an urgent need for the development of novel and effective treatments and therapeutics. LASV nucleoprotein plays a vital role in several aspects of the viral life cycle. Therefore, an effective inhibitor of LASV nucleoprotein will potentially control the replication of LASV. To evaluate the inhibitory effect of Dandelion phyto-compounds on LASV nucleoprotein, Glide-SP, and XP docking was performed for hit identification. The hit compounds were further subjected to Induced Fit Docking (IFD) followed by Prime MM-GBSA calculation and ADME studies. Dandelion phytocompounds, carfentrazone, luteolin, caffeic acid, and riboflavin recorded better binding affinity than the reference drug, ribavirin, and interacted with key amino acids residues. ADME studies also showed that our hit compounds are drug-like. This study showed that phyto-compounds of dandelion could be a better and effective therapeutics in LF treatment.
\end{abstract}

Keywords: : ADME; docking; Lassa virus; IFD; Lipinski’s rule; nucleoprotein; MM-GBSA;

Schrödinger.

(C) 2021 by the authors. This article is an open-access article distributed under the terms and conditions of the Creative Commons Attribution (CC BY) license (https://creativecommons.org/licenses/by/4.0/).

\section{Introduction}

Lassa fever (LF) is an acute and sometimes fatal viral hemorrhagic fever caused by Lassa virus (LASV), a member of the family arenaviridae and characterized by nonproductive cough, severe epigastric or retrosternal pain, and headache [1,2]. It is transmitted by an infected Mastomys natalensis, an animal commonly known as a multi-mammate rat [3], though it may also spread amid humans via direct contact with the infected person's bodily secretions, thereby contributing to its high rate of occurrence [4]. LF causes thousands of deaths annually; it is a major public health challenge and endemic exclusively in West Africa, especially in Nigeria, Benin, Togo, Guinea, Liberia, Mali, and Sierra Leone [5,6]. It is also a threat to importation 
and misuse as a bioterrorism agent in industrial countries [3]. Annually, an estimated 300,000 to 500,000 cases of LF are reported in West Africa, with circa 5,000 to 10,000 deaths [5]. Despite the large toll of human morbidity and mortality, there is no vaccine or effective drugs available to treat this disease. However, ribavirin, an antiviral agent, has been proven to be effective. It is beneficial in limiting the morbidity and mortality caused by LV if administered at an early stage of the disease [7,8]. But in a later stage, the effectiveness of ribavirin comes with potential teratogenicity and toxicity. Therefore, there is an urgent need for the development of novel and effective treatments and therapeutics.

Morphologically, LASV consists of enveloped particles that differ in diameter from circa $60 \mathrm{~nm}$ to over $300 \mathrm{~nm}$, with a mean particle size of $92 \mathrm{~nm}$ [4]. It is an enveloped virus that has two strands of single-stranded RNA. The virus has four genes and uses an ambi-sense coding approach whereby the nucleoprotein and polymerase are first transcribed. The $\mathrm{Z}$ protein and glycoprotein precursor protein are later transcribed in the life cycle after the replication of RNA [9]. The single-stranded RNA is encapsulated by the nucleoprotein, forming ribonucleoprotein (RNP), which protects the RNA from detection by the immune system [10]. LASV RNP also has an essential role in viral RNA replication and transcription. LASV Nucleoprotein consists of 569 amino acid residues comprising distinct $\mathrm{N}$ - and $\mathrm{C}$ - terminal domains connected by an unstructured linker segment. The C-terminal domain has an exonuclease to digest double-stranded RNA [11]. In contrast, the N-terminal domain is involved in RNA binding [12]. The LASV nucleoprotein plays a vital role in several aspects of the viral life cycle, including RNA encapsidation, viral replication and transcription, recruitment of ribonucleoprotein complexes to budding viral sites, and inhibition of the host cell interferon response $[12,13]$. Therefore, effective anti-nucleoprotein immunity during an early stage of the disease will potentially control the replication of LASV.

Lately, treating diseases by means of medicinal plants is capturing new interest ${ }^{\text {It }}$ has increasingly drawn attention as potential sources of antiviral drugs due to lower cost and fewer side effects. In the last years, many researchers have proved that herbs such as Taraxacum officinale, commonly known as dandelion, have considerable effects on disease treatment [14]. Dandelion is an herbaceous perennial plant of the family Asteraceae with medicinal and culinary uses [14]. Therapeutically, dandelion can eliminate toxins and heat, as well as reduce swelling, diuresis, inflammation, and choleresis [15]. According to Chinese folklore, dandelion is a treatment option for fever, lymphadenitis, acute mastitis, hepatitis, and urinary infection [15]. Studies linking specific dandelion phyto-compounds to antiviral activities are still rare and may delay drug development plans. Also, there are no side effects associated with the prolonged use of dandelion for therapeutic purposes [15]. Thus, the present study uses in silico studies via Glide (Grid-base Ligand Docking with Energetics) docking, Induced Fit Docking (IFD), Prime MM-GBSA (Molecular Mechanics-Generalized Born Surface Area), and ADME (Absorption, Distribution, Metabolism, and Excretion) to determine dandelion phytocompounds that have potential to interact with and inhibit the activity of LASV nucleoprotein in the treatment of LF.

\section{Materials and Methods}

\subsection{Protein preparation.}

The three-dimensional (3D) crystal structure of LASV nucleoprotein (PDB id: 3MX2) was downloaded from the Protein databank (www. http://www.rcsb.org/pdb). Structural 
defects of the downloaded crystal structure were fixed and were properly prepared for calculation using the protein preparation wizard of Schrödinger Maestro 11.5 [16]. Explicit hydrogen's were added to the structure; bond orders were assigned, water beyond $5.00 \AA$ was deleted, missing side chains and loops were filled using Prime. Using Epik, het states were generated at default $\mathrm{pH}$, then optimized using PROPKA at $\mathrm{pH}$ 7.0. Restrained minimization was carried out by setting heavy atom root-mean-square-deviation to $0.30 \AA$ using the OPLS3 force field. The following is the FASTA sequence format of the downloaded crystal structure of LASV nucleoprotein:

>3MX2_1|Chains A,B,C|Nucleoprotein|Lassa virus (11622)

GAMDHVEFMSASKEIKSFLWTQSLRRELSGYCSNIKLQVVKDAQALLHGLDFSEVS

NVQRLMRKERRDDNDLKRLRDLNQAVNNLVELKSTQQKSILRVGTLTSDDLLILAA DLEKLKSKVIRTERPLSAGVYMGNLSSQQLDQRRALLNMIGMSGGNQGARAGRDG VVRVWDVKNAELLNNQFGTMPSLTLACLTKQGQVDLNDAVQALTDLGLIYTAKYP NTSDLDRLTQSHPILNMIDTKKSSLNISGYNFSLGAAVKAGACMLDGGNMLETIKVS PQTMDGILKSILKVKKALGMFISDTPGERNPYENILYKICLSGDGWPYIASRTSITGRA WENTVVDLESDGKPQKADSNNSSKSLQSAGFTAGLTYSQLMTLKDAMLQLDPNAK TWMDIEGRPEDPVEIALYQPSSGCYIHFFREPTDLKQFKQDAKYSHGIDVTDLFATQP GLTSAVIDALPRNMVITCQGSDDIRKLLESQGRKDIKLIDIALSKTDSRKYENAVWDQ YKDLCHMHTGVVVEKKKRGGKEEITPHCALMDCIMFDAAVSGGLNTSVLRAVLPR DMVFRTSTPRVVL

\subsection{Ligand preparation.}

The phyto-compounds of dandelion reported by published literature were downloaded from NCBI PubChem database in two-dimensional structure (2D) and were exported to Schrödinger Maestro 11.5 for accurate and efficient 2D to 3D conversion using LigPrep [17]. A maximum of four possible stereoisomers per ligand was retained, ligands ionization states were generated at a $\mathrm{pH}$ range of $7+/-2.0$, while stereoisomers computation was left at default.

\subsection{Molecular docking.}

The active site of LASV nucleoprotein was defined based on the position and size of the co-crystallized ligand (Deoxythymidine triphosphate) using the receptor grid generation tool in Schrödinger Maestro 11.5 [16]. The prepared small molecule compounds (SMCs) were docked into the defined active site using Glide - SP (Standard Precision) and -XP (Extra Precision) docking modes embedded in the glide tool of Schrödinger Maestro 11.5 [16]. Van der Waals scaling factor and partial cutoff were left at 0.80 and 0.15 , respectively. Ultimately, induced Fit Docking (IFD) approach was carried out on the top 4 hit compounds using the IFD panel in Schrödinger Maestro 11.5. IFD introduces flexibility for both receptor and ligand during the molecular docking process.

\subsection{Molecular docking validation.}

Our molecular docking (MD) protocol was validated by calculating the root mean square deviation (RMSD) value of the crystal structure pose and docked pose of the cocrystalized ligand, deoxythymidine triphosphate. This method is the most widely used method for validating an MD protocol. 


\subsection{Prime MM-GBSA simulation.}

To calculate the binding-free energy ( $\Delta G_{\text {bind }}$ ) of each ligand-protein complex, Prime MM-GBSA was employed via Prime Schrödinger Maestro 11.5 [18], using VSGB solvation model and OPLS3 force field, while the sampling method was set as minimized. The $\Delta G_{\text {bind }}$ of each ligand with LASV nucleoprotein was calculated based on the following equation [19]:

$$
\begin{aligned}
& \Delta G_{\text {bind }}=\Delta \mathrm{E}+\Delta \mathrm{G}_{\text {solv }}+\Delta \mathrm{G}_{\text {SA }} \\
& \Delta \mathrm{E}=\mathrm{E}_{\text {complex }}-\mathrm{E}_{\text {protein }}-\mathrm{E}_{\text {ligand }}
\end{aligned}
$$

Where, $\mathrm{E}_{\text {complex }}, \mathrm{E}_{\text {protein }}$ and $\mathrm{E}_{\text {ligand }}$ are the minimized energies of the protein-inhibitor complex, protein, and inhibitor, respectively.

$$
\Delta \mathrm{G}_{\text {solv }}=\mathrm{G}_{\text {solv(complex) }}-\mathrm{G}_{\text {solv(protein) }}-\mathrm{G}_{\text {solv(ligand) }}
$$

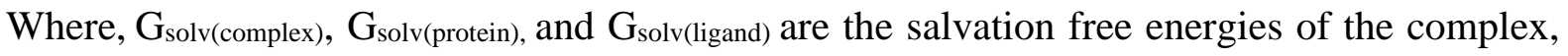
protein, and inhibitor, respectively.

$$
\Delta \mathrm{G}_{\mathrm{SA}}=\mathrm{G}_{\mathrm{SA}} \text { (complex) }-\mathrm{G}_{\mathrm{SA}} \text { (protein) }- \text { GSA(ligand) }
$$

Where GsA(complex), GsA(protein), and GsA(ligand) are the surface area energies for the complex, protein, and inhibitor, respectively.

\subsection{ADME studies.}

QikProp in Schrödinger Maestro 11.5 [20] was used to predict a variety of pharmaceutical properties of our hit compounds. Predicted ADME includes Lipinski's rule of five (RO5) violation, human oral absorption (HOA), aqueous solubility (QPlogS), human serum albumin binding (QPlogKhsa), apparent MDCK cell permeability in $\mathrm{nm} / \mathrm{sec}$ (QPPMDCK), brain/blood partition coefficient (QPlogBB), apparent Caco-2 cell permeability in $\mathrm{nm} / \mathrm{sec}$ (QPPCaco) and IC50 value for the blockage of HERG K+ channels (QPlogHERG).

\section{Results and Discussion}

\subsection{Molecular docking studies.}

MD or computer-simulated ligand binding is a structure-based approach widely used to study molecular recognition, which aims to predict the binding affinity and binding mode of a complex formed by molecules with known structures [21]. MD is a simplified form of molecular dynamics simulation [22]. In this study, the prepared phyto-compounds of dandelion were docked onto the active site of LASV nucleoprotein using Glide-SP, -XP docking, and IFD, respectively. According to this study, when dandelion phyto-compounds were docked into the active site of LASV nucleoprotein using Glide-XP docking mode, binding energy values ranged from $-10.620 \mathrm{kcal} / \mathrm{mol}$ to $-9.366 \mathrm{kcal} / \mathrm{mol}$ were obtained. Carfentrazone was ranked the highest, while riboflavin was the least in the ranking. In the same vein, ribavirin, our reference drug, was also docked into the enzyme's active site, and a binding energy value of $-7.931 \mathrm{kcal} / \mathrm{mol}$ was obtained (Table 1). Since Glide docking employs a rigid receptor's basic assumption, this may not be sufficient to treat systems where small molecule compounds (SMCs) induces significant conformational changes [23-26]. Therefore, the top 4 hit compounds with the best Glide-XP docking scores were selected for the Schrödinger IFD protocol that first uses Glide and subsequently uses Prime to thoroughly consider possible binding modes and the associated conformational changes within the active site of the target receptor. As shown in Table 1, the hit compounds; carfentrazone, luteolin, caffeic acid, and riboflavin report an IFD score of $-1140.66 \mathrm{kcal} / \mathrm{mol},-1143.08 \mathrm{kcal} / \mathrm{mol},-1139.76 \mathrm{kcal} / \mathrm{mol}$, 
and $-1142.72 \mathrm{kcal} / \mathrm{mol}$ for carfentrazone, luteolin, caffeic acid, and riboflavin, respectively. In comparison with the IFD score of ribavirin, dandelion phyto-compounds recorded stronger binding energy. Ribavirin has a low IFD score of $-1132.09 \mathrm{kcal} / \mathrm{mol}$, possibly due to its reduced penetration into the active site compared to our hit compounds. Based on these results, it is suggested that carfentrazone, luteolin, caffeic acid, and riboflavin have relatively better inhibitory activity than ribavirin.

After IFD approach, the hit compounds were well accommodated and deeply fit into the active site of LASV nucleoprotein in a similar pattern, where they bind with amino acid residues Asn240, Thr178, Lys253, Lys253, Ile241, Thr178, Arg300, Trp164, Glu117, Phe176, Trp164, Glu117, Tyr308, Asn174, Arg323, Lys309, Asn174 and Ser238 within 4 A region. As Verma et al. [4] reported and Li et al. [7], most of these residues play vital roles in the recognition and binding of substrate. From the interaction analysis of the hit compounds, it is clear that the interactions result from the formation of different bonds ( $\pi-\pi$ stacking, salt bridge, and hydrogen ( $\mathrm{H}-)$ bond interactions). Carfentrazone and luteolin form $\mathrm{H}$-bond interactions with polar residues, Asn240, and Asn173 in the case of luteolin. All compounds except luteolin also form H-bond interaction with polar residues, Thr178, and Asn174, in case of luteolin, caffeic acid, and riboflavin, as well as Ser238 and positively charged Lys253 in case of riboflavin only. Luteolin form $\mathrm{H}$-bond interactions with hydrophobic Ile241, while luteolin and caffeic acid also form $\mathrm{H}$-bond interactions with hydrophobic Trp164 and Tyr308, respectively. H-bonds play important roles in maintaining proteins' structure and forming most bio-molecular protein-ligand complexes [27]. Other molecular interactions between LASV nucleoprotein and the hit compounds include $\pi-\pi$ stacking interaction with the phenolic backbones, and salt bridge interaction between the compounds and essential amino acid residues of the protein, possibly responsible for the observed binding energy as well as the better interaction and inhibitory potential of dandelion phyto-compounds. The hit compounds form $\pi-\pi$ stacking with positively charged amino acids at the active site. Carfentrazone and caffeic acid form $\pi-\pi$ stacking interact with Arg300, while luteolin and riboflavin form $\pi$ - $\pi$ stacking interact with Phe176. Phe176 plays a pivotal role in the recognition of substrate. Ultimately, salt bridge formation was observed between the lead compounds and positively charged residues; Lys253 and Arg323. Carfentrazone and caffeic acid from salt bridge interaction with Lys253 and Arg323 in the case of carfentrazone. Interestingly, dandelion phyto-compounds displayed similar interaction with the co-crystalized ligand in terms of salt bridge formation, $\pi-\pi$ stacking, and H-bond formation, as shown in Figure 2-6, which is also consistent with the study of Li et al. [7].

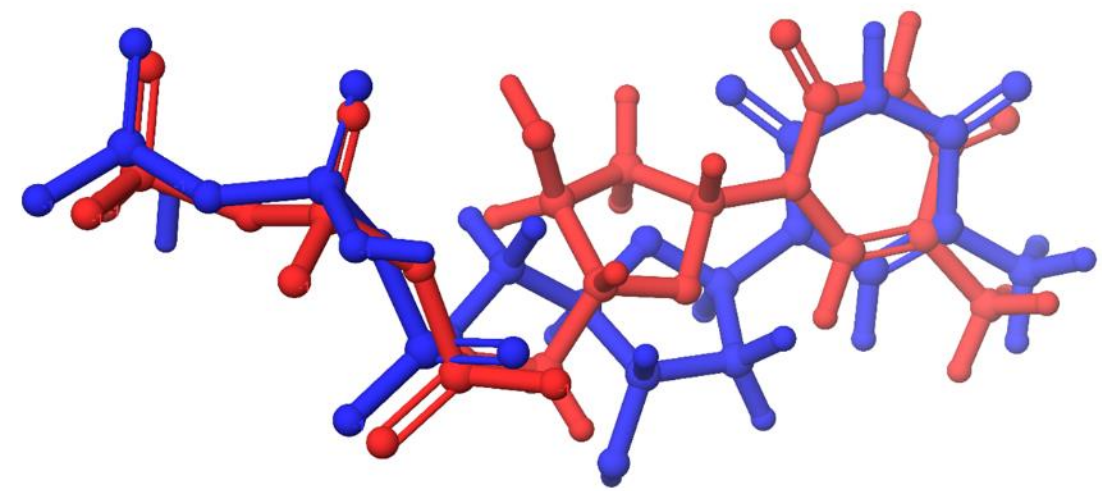

Figure 1. Validation of the molecular docking protocol employed using the before docking (blue) and after Glide-XP docking (red) pose of the co-crystallized ligand (deoxythymidine triphosphate). 


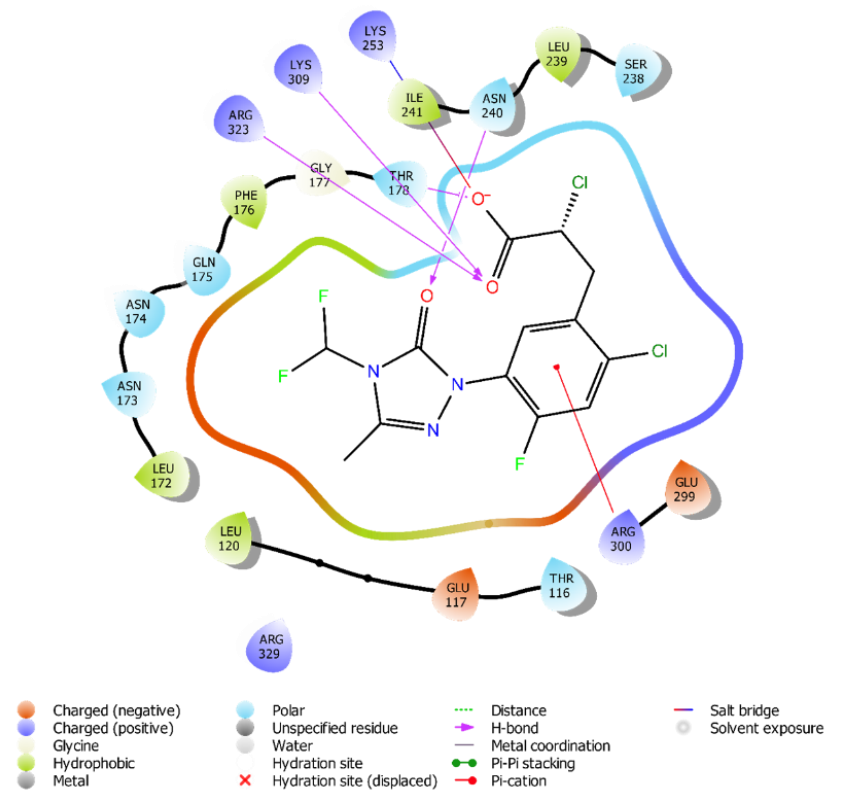

Figure 2. Interaction view of Carfentrazone with amino acid residues (4.00A) at the active site of LASV nucleoprotein upon Induced Fit Docking.
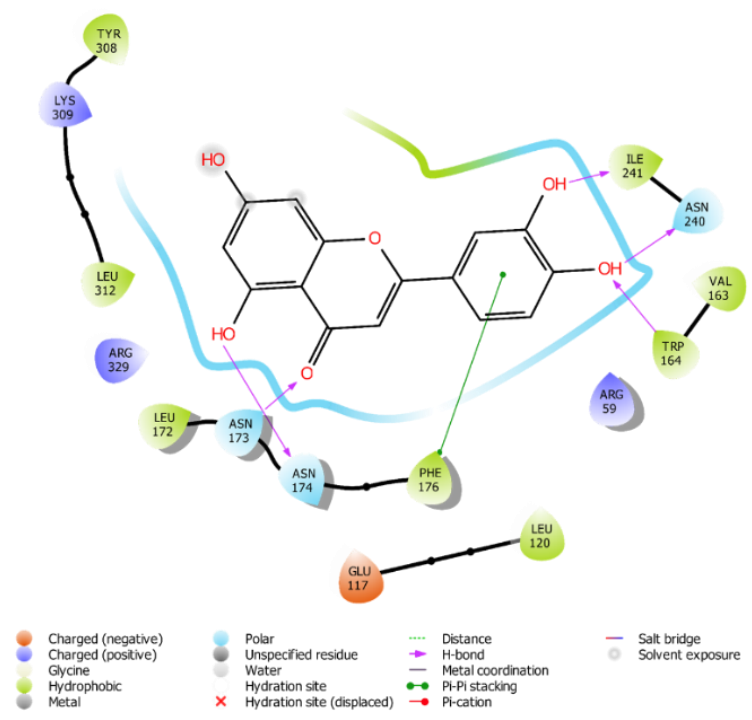

Figure 3. Interaction view of luteolin with amino acid residues $(4.00 \AA)$ at the active site of LASV nucleoprotein upon Induced Fit Docking.

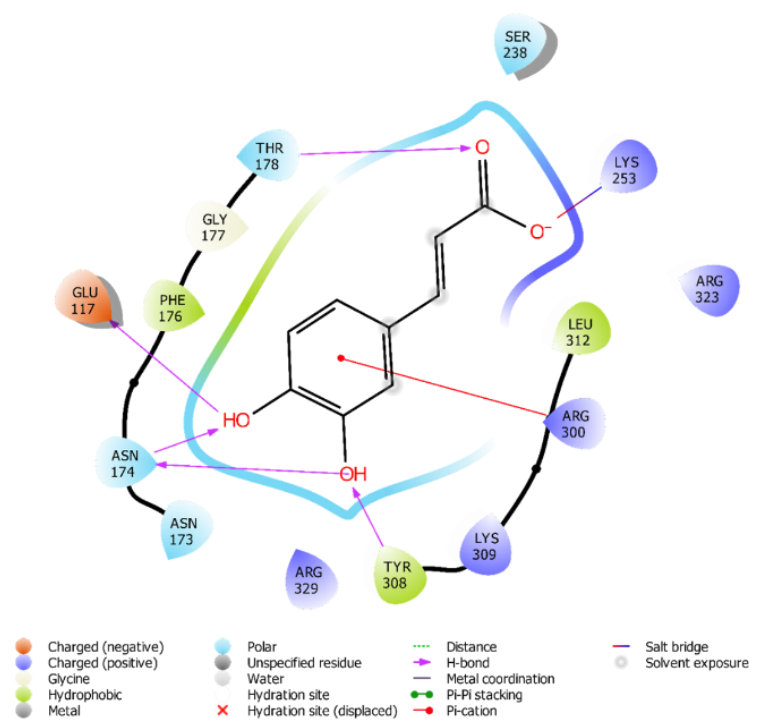

Figure 4. Interaction view of Caffeic acid with amino acid residues (4.00A) at the active site of LASV nucleoprotein upon Induced Fit Docking. 


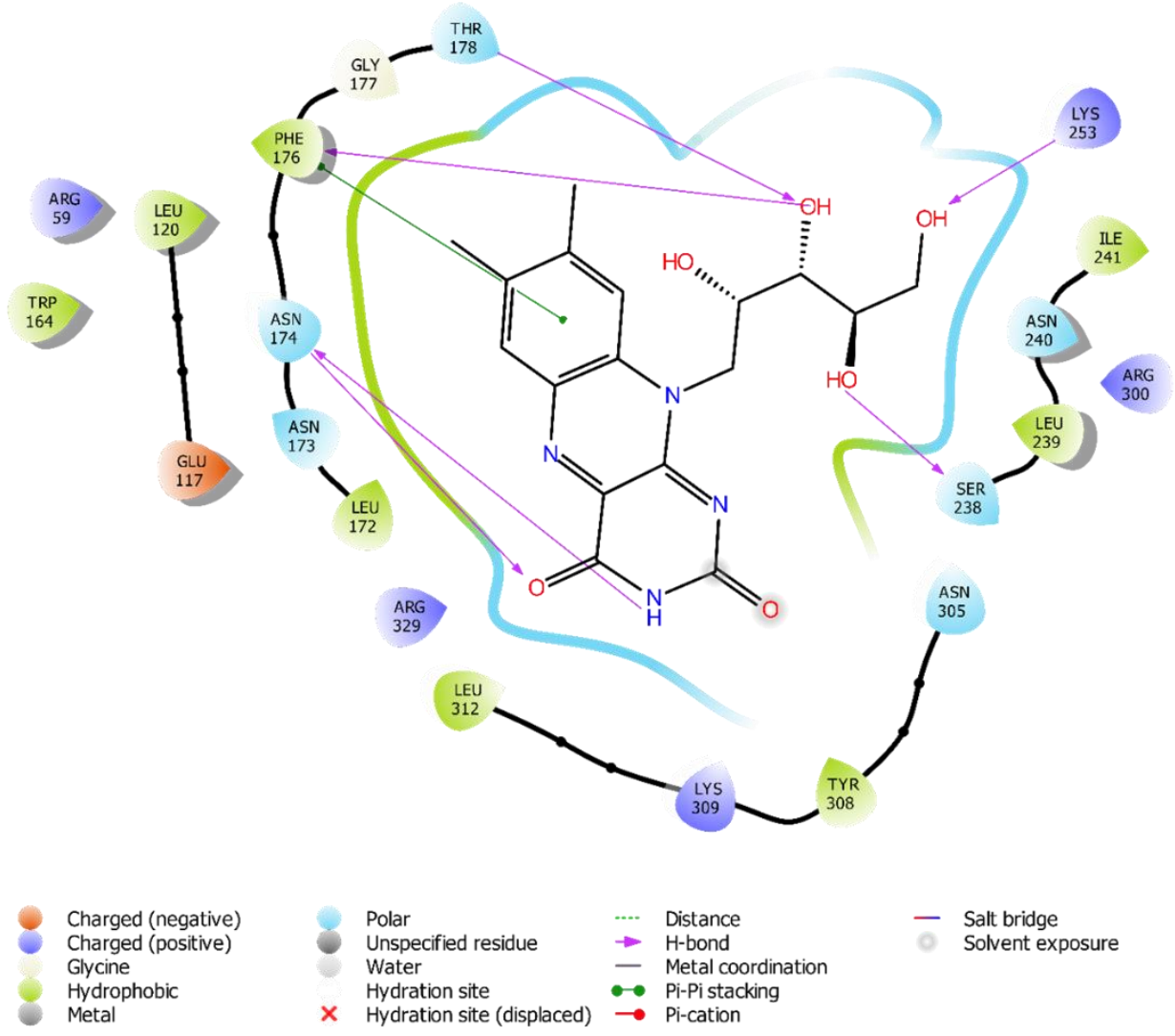

Figure 5. Interaction view of riboflavin with amino acid residues (4.00 $)$ at the active site of LASV nucleoprotein upon Induced Fit Docking.

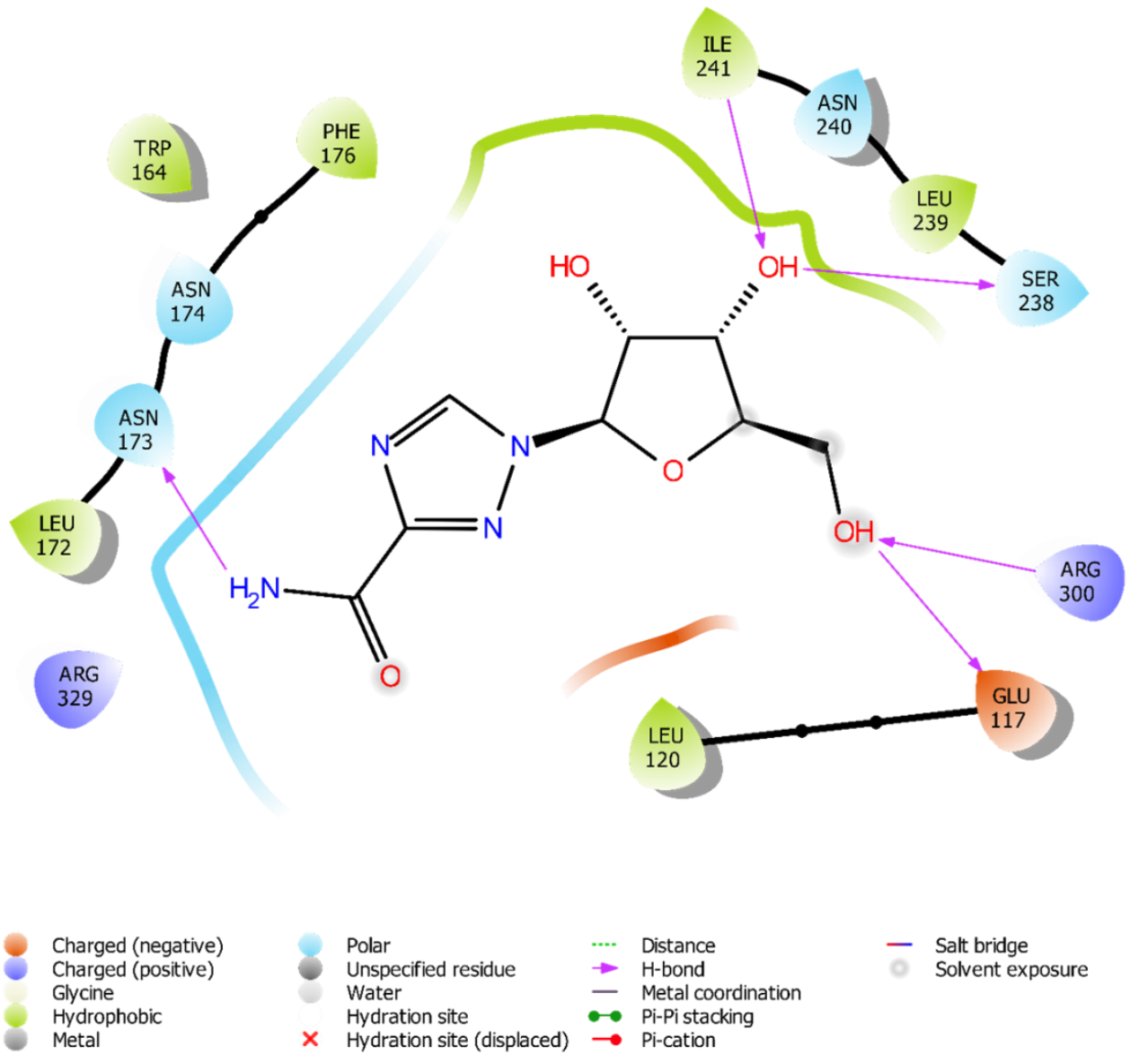

Figure 6. Interaction view of ribavirin with amino acid residues $(4.00 \AA)$ at the active site of LASV nucleoprotein upon Induced Fit Docking. 


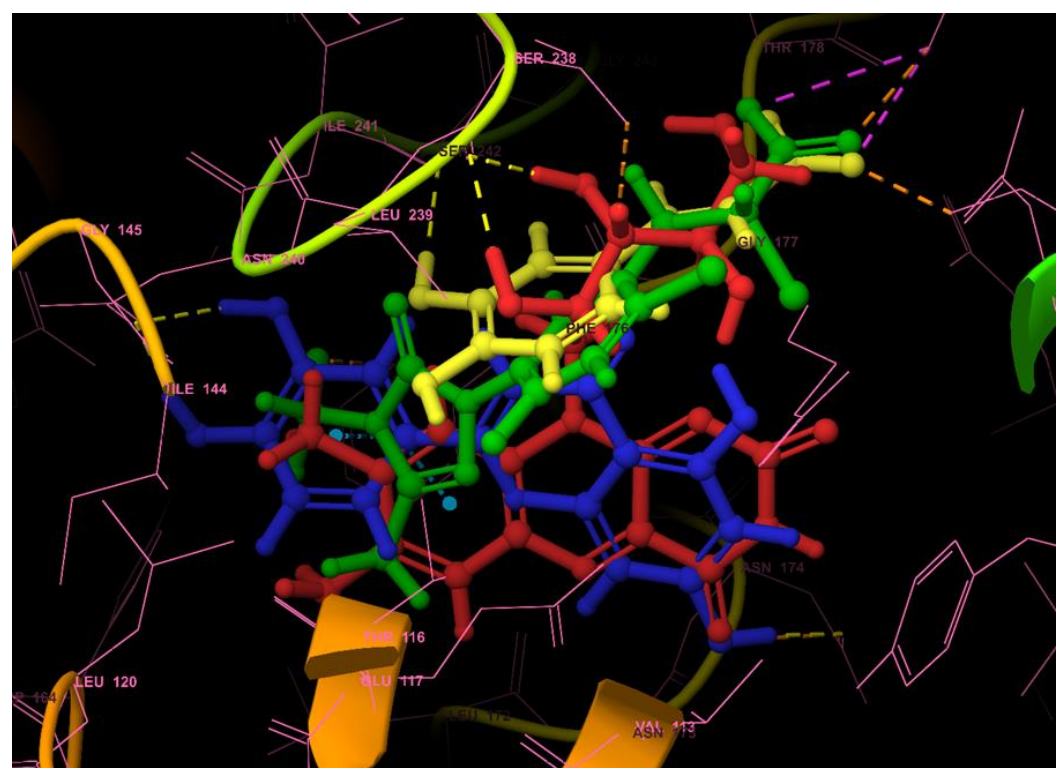

Figure 7. Three-dimensional superimposed binding pattern of the hit compounds; carfentrazone (green), luteolin (blue), caffeic acid (yellow), and riboflavin (red).

Table 1. Molecular formula, glide-XP docking, Induced Fit Docking (IFD), and binding free energy scores of the hit compounds and ribavirin.

\begin{tabular}{l|c|c|c|c}
$\begin{array}{l}\text { Dandelion phyto- } \\
\text { compounds }\end{array}$ & Molecular Formula & $\begin{array}{c}\text { PubChem } \\
\text { CID }\end{array}$ & $\begin{array}{c}\text { Glide-XP docking } \\
\text { (kcal/mol) }\end{array}$ & $\begin{array}{c}\text { IFD scores } \\
\text { (kcal/mol) }\end{array}$ \\
\hline Carfentrazone & $\mathrm{C}_{13} \mathrm{H}_{10} \mathrm{C}_{12} \mathrm{~F}_{3} \mathrm{~N}_{3} \mathrm{O}_{3}$ & 443229 & -10.620 & -1140.66 \\
\hline Luteolin & $\mathrm{C}_{15} \mathrm{H}_{10} \mathrm{O}_{6}$ & 5280445 & -9.863 & -1143.08 \\
\hline Caffeic acid & $\mathrm{C}_{9} \mathrm{H}_{8} \mathrm{O}_{4}$ & 689043 & -9.555 & -1139.76 \\
\hline Riboflavin & $\mathrm{C}_{17} \mathrm{H}_{20} \mathrm{~N}_{4} \mathrm{O}_{6}$ & 493570 & -9.366 & -1142.72 \\
\hline Apigenin & $\mathrm{C}_{15} \mathrm{H}_{10} \mathrm{O}_{5}$ & 5280443 & -8.527 & \\
\hline Esculetin & $\mathrm{C}_{9} \mathrm{H}_{6} \mathrm{O}_{4}$ & 5281416 & -8.326 & \\
\hline Sinapic acid & $\mathrm{C}_{11} \mathrm{H}_{12} \mathrm{O}_{5}$ & 637775 & -7.675 & \\
\hline Artemetin & $\mathrm{C}_{20} \mathrm{H}_{20} \mathrm{O}_{8}$ & 5320351 & -6.610 & \\
\hline Ribavirin & $\mathrm{C}_{8} \mathrm{H}_{12} \mathrm{~N}_{4} \mathrm{O}_{5}$ & & -7.931 & -1132.09
\end{tabular}

To prove our docking protocol and tools' accuracy, the co-crystalized ligand (deoxythymidine triphosphate) was extracted from the receptor protein and re-docked into the defined active site. After docking, the crystal structure poses and docked pose of deoxythymidine triphosphate were superimposed and compared in terms of RMSD. MD protocol can be considered to be accurate if the RMSD value is $\leq 2.0 \AA$ [28]. As shown in Figure 1 , the crystal structure pose and docked pose of deoxythymidine triphosphate overlapped with an RMSD value of $0.8840 \AA$, indicating a valid MD protocol. Therefore, all MD results are acceptable. The 3D superimposed binding pattern of the hit compounds are displayed in Figure 7.

\subsection{Binding-free energy analysis.}

The $\Delta G_{\text {bind }}$ for LASV nucleoprotein-hit compounds complex and LASV nucleoproteinribavirin complex were calculated using the MM-GBSA technology available with Prime; we calculated the $\Delta G_{\text {bind }}$ from the best complexes of carfentrazone, luteolin, caffeic acid, and riboflavin obtained by IFD. Studies have reported Prime MM-GBSA technology as a very reliable post docking approach for rating the affinity of SMCs on binding to their respective target receptors, and results gotten through this approach are found to be highly reproducible $[19,29-30]$. The calculated $\Delta G$ bind of LASV nucleoprotein-hit compounds complex ranged from $-88.34 \mathrm{kcal} / \mathrm{mol}$ to $-39.50 \mathrm{kcal} / \mathrm{mol}$, while LASV nucleoprotein-ribavirin complex 
recorded a $\Delta G$ bind of $-40.70 \mathrm{kcal} / \mathrm{mol}$. Thus, the $\Delta G_{\text {bind }}$ results further establish our hit compounds' stability in the active site of LASV nucleoprotein. The results of the $\Delta G_{\text {bind }}$ prediction using Prime MM-GBSA are listed in Table 2.

Table 2. Binding free energy calculation using Prime MM-GBSA approach.

\begin{tabular}{c|c|c|c|c|c|c}
$\begin{array}{c}\text { Dandelion phyto- } \\
\text { compounds }\end{array}$ & $\begin{array}{c}\Delta \text { Gbind } \\
\mathbf{( k c a l / m o l})\end{array}$ & $\begin{array}{c}\Delta \text { Gbind } \\
\text { Coulomb }\end{array}$ & $\begin{array}{c}\Delta \text { Gbind } \\
\mathbf{v d W}\end{array}$ & $\begin{array}{c}\Delta \text { Gbind } \\
\text { HBond }\end{array}$ & $\begin{array}{c}\Delta \text { Gbind } \\
\text { covalent }\end{array}$ & $\begin{array}{c}\Delta \text { Gbind } \\
\text { Lipohilic }\end{array}$ \\
\hline Carfentrazone & -60.39 & -112.88 & -46.48 & -3.61 & 1.03 & -8.97 \\
\hline Luteolin & -54.57 & -32.41 & -33.23 & -2.90 & 6.75 & -13.94 \\
\hline Caffeic acid & -39.50 & -111.45 & -14.84 & -6.79 & 0.27 & -5.70 \\
\hline Riboflavin & -88.34 & -69.78 & -48.49 & -5.26 & 6.28 & -16.89 \\
\hline Ribavirin & -40.70 & -48.66 & -26.43 & -4.71 & 3.50 & -6.29
\end{tabular}

\subsection{ADME studies.}

Nowadays, drug discovery emphasizes achieving the best possible potency against the therapeutic target of interest and in quest of compounds with good ADME properties and carrying out these two tasks in parallel, rather than in sequence. This helps to detect compounds with poor physicochemical properties at an early drug discovery stage, thereby saving both expense and time. Therefore, the evaluation of the lead compounds' physicochemical descriptors and pharmaceutically relevant properties were taken into consideration. Lipinski ROF defines the molecular properties that are essential for SMCs pharmacokinetics in the human body, including their ADME [31]. Lipinski ROF [32-33] is a rule of thumb to evaluate the drug-likeness or determine if SMCs with certain biological or pharmacological activity has physical properties or chemical properties that would make them a likely orally active drug in humans. The rule states that an orally active drug must not violate more than one of the following criteria: octanol-water partition coefficient $\leq 5$, hydrogen bond donor $\leq 5$, molecular weight $<500$ Daltons, and hydrogen bond acceptor $\leq 10$. SMCs are believed not to be a good orally administered drug candidate if they violate more than one of the abovementioned criteria [31,34]. Prospective drugs that obey the Lipinski ROF tend to have lower attrition rates during clinical trials and hence may be approved by the national regulatory authority for use in the general population $[27,35]$.

Table 3. Drug-likeness and ADME properties of the hit compounds and ribavirin based on QikProp.

\begin{tabular}{l|c|c|c|c|c|c|c|c}
$\begin{array}{l}\text { Dandelion phyto- } \\
\text { compounds }\end{array}$ & $\begin{array}{c}\text { a Rule } \\
\text { of Five }\end{array}$ & ${ }^{\mathbf{b}} \mathbf{H O A}$ & ${ }^{\mathbf{c} Q P l o g S}$ & ${ }^{\mathbf{d}}$ QPlogKhsa & eQPPMDCK & ${ }^{\text {fQPlogBB }}$ & ${ }^{\mathbf{g} Q P P C a c o}$ & hQPlogHERG \\
\hline Carfentrazone & 0 & 3 & -5.176 & 0.013 & 517.947 & -0.605 & 86.372 & -2.718 \\
\hline Luteolin & 0 & 3 & -3.063 & -0.196 & 15.963 & -1.942 & 41.721 & -5.014 \\
\hline Caffeic acid & 0 & 2 & -1.290 & -0.804 & 10.229 & -1.548 & 22.125 & -2.157 \\
\hline Riboflavin & 0 & 2 & -2.024 & -0.812 & 12.954 & -2.244 & 34.391 & -3.894 \\
\hline Ribavirin & 0 & 2 & -1.446 & -0.875 & 10.278 & -1.828 & 27.761 & -2.967
\end{tabular}

a Number of violations of Lipinski's rule of five (Range: maximum is 4); ${ }^{\mathrm{b}}$ Human Oral Absorption (Range: 3, 2 , or 1 for high, medium or low); ${ }^{c}$ Predicted aqueous solubility, $\log \mathrm{S}$. S in mol dm ${ }^{-3}$ (Range: $-8.0--1.0$ ); ${ }^{\mathrm{d}}$ Prediction of binding to human serum albumin (Range: $-1.5-1.5$ ); ${ }^{\mathrm{e}}$ Predicted apparent MDCK cell permeability in $\mathrm{nm} / \mathrm{sec}$ (Range: $<25$ poor, $>500$ great); ${ }^{\mathrm{f}}$ Predicted brain/blood partition coefficient (Range: $-3.0-1.2$ ); ${ }^{\mathrm{g}}$ Predicted apparent Caco-2 cell permeability in $\mathrm{nm} / \mathrm{sec}(<25$ poor, $>500$ great); Predicted skin permeability (Range: $-8.0--1.0)$; ${ }^{\text {h }}$ Predicted $\mathrm{IC}_{50}$ value for blockage of HERG K+ channels (Range: concern below -5 ).

Furthermore, HOA, QPlogS, QPlogKhsa, QPPMDCK, QPlogBB, QPPCaco, and QPlogHERG were also evaluated. As shown in Table 3, it is worth noting that the hit compounds conform to the Lipinski ROF, and they fall within all the recommended/accepted range. Therefore, this indicates the druggability of carfentrazone, luteolin, caffeic acid, and riboflavin.

\section{Conclusions}


This current study made a steadfast effort to identify LASV nucleoprotein inhibitors from a natural source as potential therapeutic agents in LF treatment. The result showed that phyto-compounds of dandelion, carfentrazone, luteolin, caffeic acid, and riboflavin are a possible inhibitor of LASV nucleoprotein. Also, they adhered to Lipinski ROF and other significant parameters required to be considered drug candidates. In vivo and or in vitro assays could be conducted to affirm the potency of these compounds further.

\section{Funding}

This research received no external funding.

\section{Acknowledgments}

Department of Biochemistry, Adekunle Ajasin University, Nigeria.

\section{Conflicts of Interest}

The authors declare no conflict of interest.

\section{References}

1. Hallam, S.J.; Koma, T..; Maruyama, J..; Paessler, S. Review of Mammarenavirus Biology and Replication. Frontiers in microbiology 2018, 9, https://doi.org/10.3389/fmicb.2018.01751.

2. Ba, L.; Brazil, CN.; Argentine, J.; Bolivian, M.; Venezuelan, G.; Hemorrhagic, S.; Brazil, A.N.; Trinidad, T.N.; Aa, L.; Paraguay, P.N.; Florida, T.N. Lassa, Junin, Machupo and Guanarito Viruses. Desk Encyclopedia 2010, 21.

3. Carnec, X.; Baize, S..; Reynard, S.; Diancourt, L.; Caro, V.; Tordo, N.; Bouloy, M. Lassa virus nucleoprotein mutants generated by reverse genetics induce a robust type I interferon response in human dendritic cells and macrophages. Journal of virology 2011, 85, 12093-12097, https://doi.org/10.1128/jvi.00429-11.

4. Verma, S.K.; Yadav, S.; Singh, A.; Kumar, A. Identification of Potential Inhibitor for Nucleoprotein of Lassa Virus: A Combination of Virtual Screening, ADMET Studies and Pharmacophore Modeling. J Drug Des Res 2015, 2.

5. Ogbu, O.; Ajuluchukwu, E.; Uneke, C.J. Lassa fever in West African sub-region: an overview. J Vector Borne Dis 2007, 44, 1-11.

6. Sullivan, B.M.; Sakabe, S.; Hartnett, J.N.; Ngo, N.; Goba, A.; Momoh, M.; Sandi, J.D.; Kanneh, L.; Cubitt, B.; Garcia, S.D.; Ware, B.C. High crossreactivity of human T cell responses between Lassa virus lineages. PLoS pathogens 2020, 16, https://doi.org/10.1371/journal.ppat.1008352

7. Li, L.; Li, D.; Chen, H.; Han, J.G. Studies on the binding modes of Lassa nucleoprotein complexed with $\mathrm{m} 7 \mathrm{GpppG}$ and dTTP by molecular dynamic simulations and free energy calculations. Journal of Biomolecular Structure and Dynamics 2013, 31, 299-315, https://doi.org/10.1080/07391102.2012.703061.

8. Rodriguez-Carreno, M.P.; Nelson, M.S.; Botten, J.; Smith-Nixon, K.; Buchmeier, M.J.; Whitton, J.L. Evaluating the immunogenicity and protective efficacy of a DNA vaccine encoding Lassa virus nucleoprotein. Virology 2005, 335, 87-98, https://doi.org/10.1016/j.virol.2005.01.019.

9. Yun, N.E.; Walker, D.H. Pathogenesis of Lassa fever. Viruses 2012, 4, 2031-48, https://doi.org/10.3390/v4102031.

10. Pattis, J.G.; May, E.R. Markov State Model of Lassa Virus Nucleoprotein Reveals Large Structural Changes during the Trimer to Monomer Transition. Structur 2020, 28, 548-554, https://doi.org/10.1016/j.str.2020.03.002.

11. Hastie, K.M.; Kimberlin, C.R.; Zandonatti, M.A.; MacRae, I.J.; Saphire, E.O. Structure of the Lassa virus nucleoprotein reveals a dsRNA-specific $3^{\prime}$ to $5^{\prime}$ exonuclease activity essential for immune suppression. Proceedings of the National Academy of Sciences 2011, 108, 2396-401.

12. Hastie, K.M.; Liu, T.; Li, S.; King, L.B.; Ngo, N.; Zandonatti, M.A.; Woods, V.L.; de la Torre, J.C.; Saphire, E.O. Crystal structure of the Lassa virus nucleoprotein-RNA complex reveals a gating mechanism for RNA binding. Proceedings of the National Academy of Sciences 2011, 108, 19365-70, https://doi.org/10.1073/pnas.1108515108.

13. Lennartz, F.; Hoenen, T.; Lehmann, M.; Groseth, A.; Garten, W. The role of oligomerization for the biological functions of the arenavirus nucleoprotein. Archives of virology 2013, 158, 1895-905, https://doi.org/10.1007/s00705-013-1684-9. 
14. Modaresi, M.; Resalatpour, N. The effect of Taraxacum officinale hydroalcoholic extract on blood cells in mice. Advances in hematology 2012, 2012, https://doi.org/10.1155/2012/653412.

15. He, W.; Han, H.; Wang, W.; Gao, B. Anti-influenza virus effect of aqueous extracts from dandelion. Virology Journal 2011, 8, 1-1, https://doi.org/10.1186/1743-422X-8-538.

16. Joseph, O.A.; Babatomiwa, K.; Niyi, A.; Olaposi, O.; Olumide, I. Molecular Docking and 3D QSAR Studies of C000000956 as a Potent Inhibitor of Bace-1. Drug research 2019, 69, 451-7, https://doi.org/10.1055/a0849-9377.

17. Kikiowo, B.; Ogunleye, AJ.; Inyang, OK.; Adelakun, N.S.; Omotuyi, O.I.; Metibemu, D.S.; David, T.I.; Oludoyi, O.O.; Ijatuyi, T.T. Flavones scaffold of Chromolaena odorata as a potential xanthine oxidase inhibitor: Induced Fit Docking and ADME studies. BioImpacts 2019, 10, 227-34, https://doi.org/10.34172/bi.2020.29.

18. Singh, K.D.; Muthusamy, K. Molecular modeling, quantum polarized ligand docking and structure-based 3D-QSAR analysis of the imidazole series as dual AT 1 and ET A receptor antagonists. Acta Pharmacologica Sinica 2013, 34, 1592-606, https://doi.org/10.1038/aps.2013.129.

19. Tripathi, S.K.; Muttineni, R.; Singh, S.K. Extra precision docking, free energy calculation and molecular dynamics simulation studies of CDK2 inhibitors. Journal of theoretical biology 2013, 334, 87-100, https://doi.org/10.1016/j.jtbi.2013.05.014.

20. Kikiowo, B.; Ogunleye, J.A.; Metibemu, D.S.; Omotuyi, I.O.; Adelakun, N.S. Virtual screening and pharmacokinetic studies of potential MAO-B inhibitors from traditional Chinese medicine. Journal of Biological Engineering Research and Review 2020, 7, 08-15.

21. Roy, K.; Kar, S.; Das, R.N. Understanding the basics of QSAR for applications in pharmaceutical sciences and risk assessment. Academic press 2015.

22. Zheng, L.; Alhossary, A.A.; Kwoh, C.K.; Mu, Y. Molecular dynamics and simulation. 2019; pp. 550-566.

23. Banerjee, K.; Gupta, U.; Gupta, S.; Wadhwa, G.; Gabrani, R.; Sharma, S.K.; Jain, C.K. Molecular docking of glucosamine-6-phosphate synthase in Rhizopus oryzae. Bioinformation 2011, 7, https://10.6026/007/97320630007285.

24. Du, Q.; Qian, Y.; Yao, X.; Xue, W. Elucidating the tight-binding mechanism of two oral anticoagulants to factor Xa by using induced-fit docking and molecular dynamics simulation. Journal of Biomolecular Structure and Dynamics 2019, 38, 625-633, https://doi.org/10.1080/07391102.2019.1583605.

25. Jo, S.; Kim, S.; Shin, D.H.; Kim, M.S. Inhibition of SARS-CoV 3CL protease by flavonoids. Journal of enzyme inhibition and medicinal chemistry $\mathbf{2 0 2 0}$ 35, https://doi.org/10.1080/14756366.2019.1690480.

26. Kashyap, K.; Kakkar, R. An insight into selective and potent inhibition of histone deacetylase 8 through induced-fit docking, pharmacophore modeling and QSAR studies. Journal of Biomolecular Structure and Dynamics 2019, 38, 48-65, https://doi.org/10.1080/07391102.2019.1567388.

27. Babatomiwa, K.; Joseph, O.A.; Olaposi, O.I.; Damilohun, M.S.; Niyi, A.S. Virtual Screening and Pharmacokinetic Studies of Potential MAO-B Inhibitors from Traditional Chinese Medicine. Journal of Biological Engineering Research and Review 2020, 7, 08-15.

28. Setiawan, A.A.; Kumala, S.; Dian Ratih, L.; Yuliana, N.D. In Silico Study On S-Allyl Cysteine and Quercetin from Garlic (Allium sativum Linn) As Xanthine Oxidase Inhibitor. SemanticScholar 2019.

29. Maffucci, I.; Hu, X.; Fumagalli, V.; Contini, A. An efficient implementation of the Nwat- MMGBSA method to rescore docking results in medium-throughput virtual screenings. Front Chem 2018, 6, https://doi.org/10.3389/fchem.2018.00043.

30. Iwaloye, O.; Elekofehinti, O.O.; Oluwarotimi, E.A.; Iwa Kikiowo, B.; Fadipe, T.M. Insight into glycogen synthase kinase- $3 \beta$ inhibitory activity of phyto-constituents from Melissa officinalis: in silico studies. In Silico Pharmacology. 2020, 8, 1-3, https://doi.org/10.1007/s40203-020-00054-X.

31. Lipinski, C.A.; Lombardo, F.; Dominy, B.W.; Feeney, P.J. Experimental and computational approaches to estimate solubility and permeability in drug discovery and development settings. Adv Drug Deliv Rev 2001, 46, 3-26, https://doi.org/10.1016/s0169-409x(00)00129-0.

32. Kikiowo, B.; Ogunleye, J.A.; Iwaloye, O.; Ijatuyi, T.T. Therapeutic potential of Chromolaena odorata phytoconstituents against human pancreatic $\alpha$-amylase. Journal of Biomolecular Structure and Dynamics 2020, 14, 1-2, https://doi.org/10.1080/07391102.2020.1833758.

33. Iwaloye, O.; Elekofehinti, O.O.; Momoh, A.I.; Babatomiwa, K.; Ariyo, E.O. In silico molecular studies of natural compounds as possible anti-Alzheimer's agents: ligand-based design. Network Modeling Analysis in Health Informatics and Bioinformatics 2020, 9, 1-4, https://doi.org/10.1007/s13721-020-00262-7.

34. Iwaloye, O.; Elekofehinti, O.O.; Babatomiwa, K.; Fadipe, T.M. In silico molecular studies of selected compounds as novel inhibitors for phosphodiesterase-5 (PDE5) in the management of erectile dysfunction. J Comput Sci Syst Biol 2020, 13, https://doi.org/10.37421/jcsb.2020.13.307.

35. Agarwal, P.; Ishida, K.; Reid, D.L.; Gupta, A. Clearance prediction for Amgen molecules against Extended Clearance Classification System (ECCS) and future directions. Drug Discovery Today 2020, https://doi.org/10.1016/j.drudis.2020.10.008. 